\title{
Ostemyelitis of the clivus due to Fusobacterium necrophorum
}

\author{
Amir Fallahian, Neal Desai, Mary Anne Jackson \\ Jean Claude Decarrie, Douglas C Rivard
}

\begin{abstract}
Introduction: Septic jugular thrombosis with secondary osteomyelitis of the clivus due to Fusobacterium necrophorum is a rare complication of Lemierre's syndrome. Other complications that can occur include cavernous sinus thrombosis and cerebral arterial vasospasm. Case Report: We present a case of a young girl illustrating the clinical findings of Lemierre's syndrome in addition to other complications that may occur. Retropharyngeal infection with internal jugular venous thrombosis was present in this case along with Fusobacterium necrophorum septicemia. Lemierre's syndrome consists of oropharyngeal infection with internal jugular
\end{abstract}

Amir Fallahian ${ }^{1}$, Neal Desai ${ }^{2}$, Mary Anne Jackson ${ }^{3}$, Jean Claude Decarrie ${ }^{4}$, Douglas C. Rivard ${ }^{5}$

Affiliations: ${ }^{1}$ Radiologist, Department of Radiology, University of Missouri, Columbia, MO, USA; ${ }^{2}$ Medical Student, University of Missouri-Kansas City School of Medicine, Kansas City, MO, USA; ${ }^{3}$ Professor of Pediatrics, Department of Infectious Disease, Children's Mercy Hospital and Clinics and the University of Missouri-Kansas City School of Medicine, Kansas City, MO, USA; ${ }^{4}$ Radiologist, CHU Ste-Justine, Imagerie Medicale, Montreal, Quebec, Canada; ${ }^{5}$ Assistant Professor of Radiology, Department of Radiology, Children's Mercy Hospital and Clinics and the University of Missouri-Kansas City School of Medicine, Kansas City, MO, USA.

Corresponding Author: Douglas C. Rivard, DO, Childrens Mercy Hospital and Clinics, Department of Radiology, 2401 Gillham Rd, Kansas City, MO 64108; Phone: 1816-234-3270; Email: dcrivard@cmh.edu

Received: 21 December 2011

Accepted: 04 March 2011

Published: 01 July 2011 vein thrombosis, anaerobic septicemia and septic embolization to multiple organ systems. Osteomyelitis of the clivus is a rare, but important complication associated with this syndrome. Fusobacterium necrophorum, the usual pathogen implicated in Lemierre's syndrome has rarely been known to be the sole pathogen implicated in childhood osteomyelitis secondary to septic jugular vein thrombosis. It is important to recognize some of the complications of septic jugular vein thrombosis to reduce morbidity and mortality. Conclusion: Awareness of the clinical and radiologic findings that may be seen in pediatric retropharyngeal infections along with awareness of the possible complications can help guide early treatment or surgical intervention.

Keywords: Osteomyelitis, Clivus, Fusobacterium Necrophorum, Lemierre's syndrome

$* * * * * * * * *$

Fallahian A, Desai N, Jackson MA, Decarrie JC, Rivard DC. Ostemyelitis of the clivus due to Fusobacterium Necrophorum. International Journal of Case Reports and Images 2011;2(7):17-20.

$$
* * * * * * * * *
$$

doi:10.5348/ijcri-2011-07-45-CR-5

\section{INTRODUCTION}

Septic jugular vein thrombosis was described as early as 1900 , but Lemierre published the first case series in 1936 [1]. Lemierre's syndrome as originally 
described included oropharyngeal infection, suppurative thrombophlebitis, anaerobic septicemia, and septic embolization to multiple organ systems [1]. It is usually caused by the bacterium, Fusobacterium necrophorum. Lemierre's syndrome is less common today, but increased awareness of this diagnosis by physicians can help facilitate early treatment, thus avoiding significant morbidity and mortality.

We present a case of a pediatric patient presenting with osteomyelitis of the clivus due to Fusobacterium necrophorum documented by cultures from their CSF, blood and surgical specimens. Venous thrombosis, septicemia and clivus osteomyelitis were present. This case illustrates clival involvement by Fusobacterium necrophorum which is a rare complication of Lemierre's syndrome.

\section{CASE REPORT}

A 9-year-old girl presented with four days of progressive fever, chills and sore throat. She also had neck pain and stiffness that developed over the four day course of constitutional symptoms. The patient was ataxic and lethargic at the time of presentation. The physical exam and laboratory findings were remarkable for medial and downward deviation of the right eye and leukocytosis.

Computed tomography (CT) scan with contrast showed retroadenoidal and retropharyngeal abscess with homogeneous enhancement of the cavernous sinus consistent with thrombosis, in addition to several foci of air in the clivus (Figure 1 A, B). Magnetic resonance imaging (MRI) and MR venography (MRV) confirmed the retroadenoidal and retropharyngeal abscess and demonstrated subperiosteal fluid dorsal to the clivus (Figures 2, 3). Abnormal signal involving the marrow of the clivus in conjunction with the air seen on CT scan and the adjacent abscess were suggestive of osteomyelitis.

The patient underwent adenoidectomy and surgical drainage of the retroadenoidal and retropharyngeal abscesses. Surgical cultures were positive for Fusobacterium necrophorum and she was initially treated with vancomycin, ceftriaxone, metronidazole, and intravenous penicillin. Anticoagulation therapy was also initiated. Following a second drainage procedure of retropharyngeal abscess three days later, her antimicrobial coverage was reduced to intravenous penicillin but was then changed once susceptibilities returned completing therapy with oral clindamycin $225 \mathrm{mg}$ P.O. every eight hours and metronidazole 250 mg every six hours; a total of 68 total days of antimicrobial therapy including 61 days of metronidazole. After 17 days of inpatient treatment, the patient was discharged without recurrence or morbidity. At follow up four years later, she had no evidence of squeal.


Figure 1: A) Contrast-enhanced neck CT scan - Sagittal reformatted soft tissue image shows retro-adenoidal (arrow) and retro-pharyngeal (arrowhead) abscesses, B) Contrastenhanced neck CT - Sagittal bone window shows gas in the clivus suggestive of osteomyelitis.

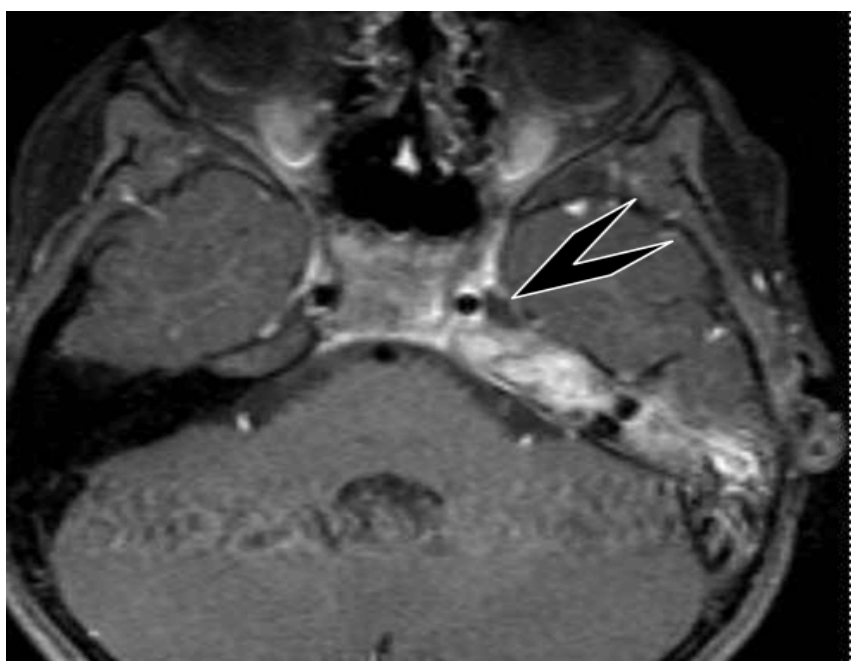

Figure 2: T1 axial fat suppressed MRI with contrast enhancement confirms inflammatory process of the left temporal bone and shows a small thrombus in the left cavernous sinus (arrowhead). 


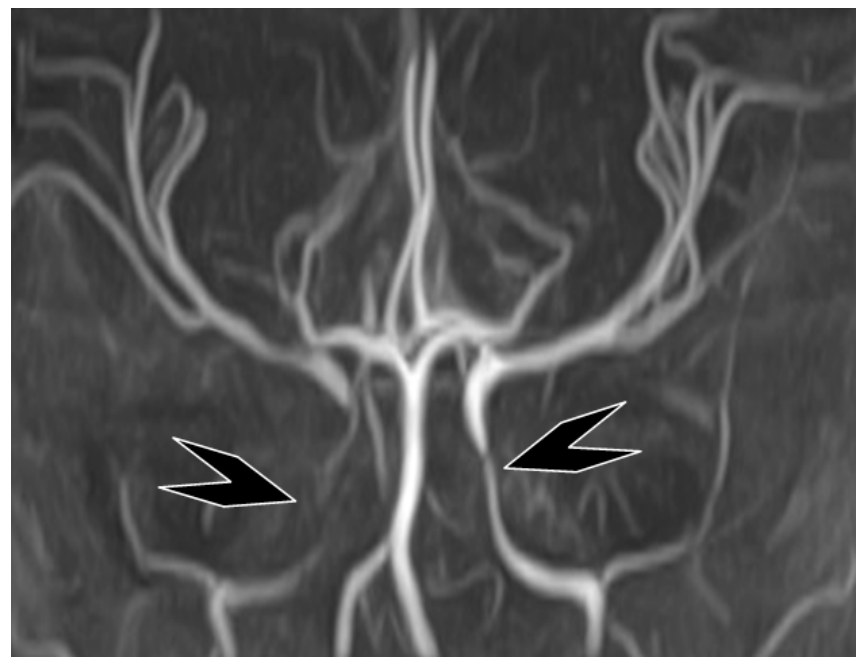

Figure 3: Magnetic Resonance Angiography brain shows absent right and narrow left (arrowheads) internal carotid arteries.

\section{DISCUSSION}

Fusobacterium species have been recovered in a variety of infections, usually as co-pathogens, including abscesses, aspiration pneumonia, paronychia, chronic sinusitis and otitis. Skeletal septic metastasis is a well known complication of jugular septic thrombophlebitis, but Fusobacterium has rarely been reported as the sole pathogen in childhood osteomyelitis as was the case in the patient presented here.

Fusobacterium necrophorum is a gram-negative, obligate, anaerobic bacillus found in oral flora and in the gastrointestinal tract. Typical infection follows pharyngitis or tonsillitis with disruption of the mucosa allowing for egress of bacteria into the parapharyngeal and peritonsillar veins. Septic phlebitis and ultimately thrombosis result, resulting in a source for dissemination of septic emboli to distant sites including the skeleton, liver and lungs. Septicemia usually follows initial infection by 4-8 days although cases have been reported where the interval between infection and septicemia was as long as two weeks [2]. When present, clinical findings of jugular thrombosis include lateral neck or jaw pain on the affected side. Current therapy has reduced the mortality rate from 90\% of patients presenting with Lemierre's syndrome to $4-18 \%$ today [3].

The case presented illustrates the high variability in the severity of the complications arising from the ubiquitous clinical entity of pharyngitis. Due to the close proximity of the clivus to the cavernous sinus and cerebral arterial vasculature at the base of the skull, additional complications secondary to osteomyelitis of the clivus can occur as well. In our case involvement of the cavernous sinus resulted in cavernous sinus thrombosis. Also possible is infarction in the distribution of the middle cerebral artery due to carotid artery being occluded by vasospasm. Although these complications are less likely in the era of antibiotics, this case illustrates the importance of high clinical suspicion in patients presenting with pharyngitis with associated symptoms of systemic disease or neurologic deficits. The antimicrobial therapy in this case was specific to cover for sensitivities from surgical cultures. Broad spectrum antimicrobial coverage would be required if a culture could not be obtained.

Other diseases can present with involvement of the clivus and may be considered in the differential diagnosis. Primary or metastatic lymphoma or leukemia can occur in the clivus, but those are usually distinguished by different magnetic resonance imaging findings. Leukemic involvement frequently results in osteolysis and adjacent soft tissue mass or periostitis [4]. Metastatic neuroblastoma is also a consideration in the pediatric patient, but clival involvement is less common than orbital or calvarial involvement [5]. Chordoma of the clivus is rare in children, but if it does occur, it is more likely to be located in the skull base at the sphenoid-occipital synchondrosis, unlike adults where a sacral location predominates. Chordoma is typically highly desctructive and heterogenous in appearance [6]. The presence of normally enhancing marrow in the infant skull base makes it more challenging in some cases to discern osteomyelitis on the basis of imaging features alone, which is why the clinical history is extremely important. The presence of gas in the clivus without a history of trauma, however does allow for fairly confident diagnosis of osteomyelitis from a gas forming organism [7].

\section{CONCLUSION}

Lemierre's Syndrome is a well known clinical entity caused by Fusobacterium necrophorum. When faced with a patient with an oropharyngeal infection along with systemic or neurologic deficits, it is important to consider the rare, but important complications such as osteomyelitis of the clivus secondary to septic jugular thrombosis. Increased awareness of these rare complications is necessary to help facilitate early diagnosis and treatment thus avoiding significant morbidity and mortality.

$$
* * * * * * * * *
$$

\section{Author Contributions}

Amir Fallahian - Conception and design, Acquisition of data, Analysis and interpretation of data, Drafting the article, Critical revision of the article, Final approval of the version to be published 
Neal Desai - Analysis and interpretation of data, Drafting the article, Critical revision of the article, Final approval of the version to be published

Mary Anne Jackson - Group 1 - Analysis and interpretation of data, Drafting the article, Critical revision of the article, Final approval of the version to be published

Jean Claude Decarrie - Acquisition of data, Analysis and interpretation of data, Drafting the article, Final approval of the version to be published

Douglas C. Rivard - Conception and design, Acquisition of data, Analysis and interpretation of data, Drafting the article, Critical revision of the article, Final approval of the version to be published

\section{Guarantor}

The corresponding author is the guarantor of submission.

\section{Conflict of Interest}

Authors declare no conflict of interest.

\section{Copyright}

(C) Douglas C. Rivard et al. 2011; This article is distributed under the terms of Creative Commons attribution 3.0 License which permits unrestricted use, distribution and reproduction in any means provided the original authors and original publisher are properly credited. (Please see www.ijcasereportsandimages.com /copyright-policy.php for more information.)

\section{REFERENCES}

1. Lemierre A. On Certain Septicaemias Due To Anaerobic Organisms. Lancet. 1936 March:701-3.

2. Sinave CP, Hardy GJ, Fardy PW. The Lemierre Syndrome: Suppurative Thrombophlebitis of the Internal Jugular Vein Secondary to Oropharyngeal Infection. Medicine. 1989;68:85-94.

3. Hagelskjaer LH. Eur J Clin Microbiol Infect Dis. 1936;17:561-5.

4. Guermazi A, Rousselot $\mathrm{P}$, Merad $\mathrm{M}$ et al. Granulocytic Sarcoma (Chloroma): Imaging Findings in Adults and Children. AJR. 2002;178:319-25.

5. Papaioannou G, McHugh K. Neuroblastoma in Childhood: Review and Radiological Findings. Cancer Imaging. 2005;5:116-27.

6. Wippold FJ $2^{\text {nd }}$, Koeller KK, Smirniotopoulos JG. Clinical and Imaging Features of Cervical Chordoma. AJR. 1999;172:1423-6.

7. Murray ME, Britton J. Osteomyelitis of the Skull Base: The Role of High Resolution CT in Diagnosis. Clin Radiol. 1994;49(6):408-11. 